\title{
РЕАКТИВНОСТЬ $\mu$-РИТМА ЭЭГ У ДЕТЕЙ ПРИ ИМИТАЦИИ ДВИЖЕНИЙ ВИЗУАЛЬНЫХ ОБРАЗОВ БИОЛОГИЧЕСКОГО И НЕБИОЛОГИЧЕСКОГО ПРОИСХОЖДЕНИЯ
}

\author{
А. И. Кайда $\bowtie$, А. А. Михайлова, Е. В. Эйсмонт, Л. Л. Джаппарова, В. Б. Павленко
}

Крымский федеральный университет имени В. И. Вернадского, Симферополь, Россия

\begin{abstract}
В настоящее время актуальна разработка интерфейсов мозг-компьютер, основанных на использовании параметров реактивности сенсомоторных ритмов ЭЭГ и предназначенных для реабилитации людей с нарушениями двигательных функций, в том числе детей. Целью работы было проанализировать реактивность $\mu$-ритма ЭЭГ в индивидуально определенном частотном диапазоне у детей при имитации движений визуальных образов биологического и небиологического происхождения. ЭЭГ регистрировали во фронтальных, центральных и париетальных областях коры у 136 нормально развивающихся детей-правшей 4-15 лет в состоянии двигательного покоя, а также при самостоятельном выполнении и имитации движений с помощью компьютерной мыши. При выполнении детьми самостоятельных движений компьютерной мышью $\left(F_{1,132}=31,17 ; p<0,001\right)$ и при осуществлении концентрических перемещений цветного круга $\left(F_{1,132}=90,34 ; p<0,001\right)$ развивается десинхронизация $\mu$-ритма во фронтальных, центральных и париетальных областях неокортекса. При имитации движений визуальных образов небиологического происхождения, по сравнению с заданием на выполнение самостоятельных движений, была выявлена синхронизация $\mu$-ритма $\left(F_{1,132}=12,65 ; p<0,001\right)$. При подражании движениям визуальных образов биологического происхождения относительно самостоятельных движений выявлена десинхронизация $\mu$-ритма $\left(F_{1,132}=9,58\right.$; p = 0,002). Данные эффекты имели свои особенности в группах детей 4-6, 7-9, 10-12 и 13-15 лет. Результаты исследования показывают целесообразность учета возрастных особенностей реактивности н-ритма и характера предъявляемых зрительных стимулов при разработке программного обеспечения интерфейсов мозг-компьютер.
\end{abstract}

Ключевые слова: дети; ЭЭГ; н-ритм; имитация; биологическое движение; небиологическое движение

Финансирование: исследования выполнены в рамках темы: «Разработка комплекса экзоскелета кисти с внешним программным управлением и биологической обратной связью для процедуры реабилитации детей с синдромом ДЦП» при финансовой поддержке Министерства науки и высшего образования Российской Федерации (RFMEFI60519Х0186).

Вклад авторов: А. И. Кайда - набор и обработка данных, написание статьи; А. А. Михайлова - обработка данных, написание статьи; Е. В. Эйсмонт план исследований, набор и обработка данных, написание статьи; Л. Л. Джаппарова — набор данных, написание статьи; В. Б. Павленко - план исследований, обработка данных, написание статьи.

Соблюдение этических стандартов: исследование одобрено этическим комитетом ФГАОУ ВО «Крымский федеральный университет им. В. И. Вернадского» (протокол № 12 от 14 июня 2016 г.). Получено добровольное информированное согласие родителей на участие детей в эксперименте.

$\triangle$ Для корреспонденции: Анна Ивановна Кайда ул. Ангарская, д. 38, г. Симферополь, 295001; kaydaanna@gmail.com

Статья получена: 01.04.2020 Статья принята к печати: 15.04.2020 Опубликована онлайн: 16.04.2020

DOI: $10.24075 /$ vrgmu.2020.019

\section{EEG $\mu$-RHYTHM REACTIVITY IN CHILDREN DURING IMITATION OF BIOLOGICAL AND NON-BIOLOGICAL MOTION}

\author{
Kaida AI $\bowtie$, Mikhailova AA, Eismont EV, Dzhapparova LL, Pavlenko VB
}

V.I. Vernadsky Crimean Federal University, Simferopol, Russia

The development of brain-computer interfaces based on the use of EEG sensorimotor rhythms reactivity parameters and designed for the rehabilitation of people (including children) with impaired motor functions is currently relevant. The study was aimed to analyse the EEG $\mu$-rhythm in the individual frequency range in children during imitation of biological and non-biological motion. EEG was recorded at frontal, central and parietal cortical regions in 136 normally developing right-handed children aged 4-15, at rest and during the execution and imitation of movements using the computer mouse. When the children moved the computer mouse on their own $\left(F_{1,132}=31.17 ; p<0.001\right)$ and executed the concentric moving of the coloured circle $\left(F_{1,132}=90.34 ; p<0.001\right)$, the $\mu$-rhythm desynchronization developed in the frontal, central and parietal neocortical regions. The $\mu$-rhythm synchronization was detected during the non-biologocal motion imitation $\left(F_{1,132}=12.65 ; p<0.001\right)$, compared to the task on the autonomous movement execution. The $\mu$-rhythm desynchronization was observed during the biologocal motion imitation in relation to autonomous movement execution $\left(F_{1,132}=9.58 ; p=0.002\right)$. The described effects had their own features in the groups of children aged $4-6,7-9,10-12$ and $13-15$. The study results demonstrate the desirability of taking into account the $\mu$-rhythm reactivity age-related features and the visual stimuli nature when developing software for the brain-computer interfaces.

Keywords: children; EEG; $\mu$-rhythm; imitation; biological motion; non-biological motion

Funding: the study was performed as a part of the project "Development of a complex of exoskeleton, hands and procedures for the rehabilitation of children with cerebral palsy" supported by the Ministry of Science and Higher Education of Russian Federation (RFMEFI60519X0186).

Author contribution: Kaida $\mathrm{Al}$ — data acquisition and analysis, manuscript writing; Mikhailova AA — data analysis, manuscript writing; Eismont EV — research planning, data acquisition and analysis, manuscript writing; Dzhapparova LL — data acquisition, manuscript writing; Pavlenko VB — research planning, data analysis, manuscript writing.

Compliance with ethical standards: the study was approved by the Ethics Committee of V.I. Vernadsky Crimean Federal University (protocol № 12 dated June 14, 2016). Informed consent to participation in the study was obtained from the parents.

$\triangle$ Correspondence should be addressed: Anna I. Kaida Angarskaya, 38, Simferopol, 295001; kaydaanna@gmail.com

Received: 01.04.2020 Accepted: 15.04.2020 Published online: 16.04.2020

DOI: $10.24075 /$ brsmu.2020.019

Способность человека понимать цели действий и имитировать их играет важную роль в эффективной интеграции в социальную среду, позволяя овладевать различными видами деятельности и нормами поведения 
в социуме. В процессах распознавания движений и связанных с ними намерений значительную роль играет система зеркальных нейронов $(\mathrm{C} 3 \mathrm{H})$ - нервных клеток, способных активироваться сходным образом, как при самостоятельном выполнении действий, так и при наблюдении за аналогичными действиями, выполняемыми другими индивидами [1, 2]. Было выдвинуто предположение, что СЗН играет важную роль в реализации сложных форм социального взаимодействия [3].

Маркером активации СЗН у человека принято считать десинхронизацию сенсомоторного ритма, или н-ритма, ЭЭГ [4]. Поскольку на эффекты десинхронизации н-ритма могут накладываться модуляции затылочного $\alpha$-ритма [5], для определения его индивидуального частотного диапазона и оценки реактивности учитывают следующие особенности: в отличие от затылочного $\alpha$-ритма, $\mu$-ритм наиболее выражен во фронто-париетальных областях; его амплитуда падает при движениях субъекта, их представлении или при наблюдениях за движениями окружающих, но существенно не изменяется при открывании или закрывании глаз [6, 7].

Подавление амплитуды сенсомоторного ритма во время наблюдения за биологическим движением более выражено, чем при наблюдении небиологического движения [8], что также характерно для наблюдения социальных действий, по сравнению с действиями вне социального контекста [9]. Что касается подражания движениям, то предполагают, что оно сопровождается активацией СЗН человека и является результатом сопоставления наблюдаемого действия и внутреннего моторного плана осуществления этого действия [10].

Исследование СЗН и реактивности н-ритма вызывает особый интерес в свете разработки новых методов реабилитации пациентов с различными двигательными нарушениями с помощью интерфейсов мозг-компьютер $[11,12]$. В частности, при лечении взрослых пациентов применяют синхронные интерфейсы, основанные на анализе реактивности сенсомоторных ритмов ЭЭГ при представлении движения в ответ на предъявляемый сигнал $[13,14]$. В последнее время такие методы начинают использовать в процессе реабилитации детей с синдромом детского церебрального паралича (ДЦП) [15]. В качестве предъявляемых пациентам сигналов в указанных работах используют символы либо текстовые команды. Однако с учетом концепции СЗН можно предположить, что более эффективными для вызова реакций в диапазоне $\mu$-ритма ЭЭГ будут стимулы, наглядно представляющие движения и требующие их имитации. Следует отметить, что при работе с детьми для более эффективной реализации задания предпочтительно использовать действия, находящиеся в моторном репертуаре ребенка [16]. В связи с вышеизложенным, целью настоящей работы было проанализировать реактивность $\mu$-ритма ЭЭГ у детей 4-15 лет в условиях имитации движений визуальных образов биологического и небиологического происхождения с помощью компьютерного манипулятора - мьши. Действия с компьютерной мышью в настоящее время хорошо знакомы даже детям дошкольного возраста.

\section{ПАЦИЕНТЫ И МЕТОДЫ}

\section{Характеристика выборки}

Исследование проводили на базе Центра коллективного пользования научным оборудованием «Экспериментальная физиология и биофизика» ФГАОУ ВО «КФУ имени В. И. Вернадского».

В исследовании приняли участие 136 детей-правшей в возрасте 4-15 лет (69 мальчиков и 67 девочек). Критерии включения в эксперимент: наличие нормального (или скорректированного до нормального) уровня зрения и слуха; предпочтение правой руки в работе с компьютерной мышью; достаточный уровень когнитивного развития (IQ не менее 80 баллов по тесту Векслера в вариантах WPPSI и WISC). Критерии исключения: прием препаратов, влияющих на работу ЦНС; тяжелые хронические соматические заболевания. В дальнейшем дети были разделены на четыре возрастные группы: 4-6 лет (30 человек), 7-9 лет (46 человек), 10-12 лет (30 человек) и 13-15 лет (30 человек).

\section{Регистрация ЭЭГ}

Регистрацию ЭЭГ осуществляли с помощью электроэнцефалографа «Нейрон-Спектр-3» («Нейрософт»; Россия). Данные обрабатывали с помощью компьютерной программы «WinEEG» версии 2.8 (в свободном доступе). Для коррекции артефактов применяли метод независимых компонент. ЭЭГ-потенциалы отводили монополярно в 19 отведениях по системе 10-20. Областью интересов в данном исследовании были фронтальные, центральные и париетальные зоны неокортекса (локусы F3, F4, Fz, C3, C4, Cz, P3, P4, Pz). Референтным электродом служили объединенные контакты, закрепленные на мочках ушей. Частоты среза фильтров высоких и низких частот составляли, соответственно, 1,5 и 35 Гц, частота оцифровки ЭЭГ-сигналов - 250 Гц.

Запись ЭЭГ производили во время выполнения испытуемыми серии последовательных заданий длительностью по 30 с каждое. Отрезки ЭЭГ подвергали быстрому преобразованию Фурье с эпохой анализа 4 с и взаимным перекрытием эпох 50\%.

В ситуации имитации движений визуальных образов небиологического происхождения использовали следующие задания:

1) фиксация взгляда на видеоизображении компьютерной мыши (фон);

2) осуществление концентрических перемещений цветного круга на экране монитора при помощи компьютерной мыши (Дв.1) (рис. 1А);

3) имитация движений другого цветного круга (имитация небиологического движения, ИмНБ) (рис. 1Б).

В ситуации имитации движений визуальных образов биологического происхождения испытуемый и экспериментатор располагались за стоящими рядом столами (экспериментатор справа), на каждом из которых находились монитор и компьютерная мышь. На мониторе перед испытуемым с помощью вебкамеры демонстрировали рабочую плоскость стола экспериментатора с расположенной на ней мышью. Последовательность заданий была следующая:

1) фиксация взгляда на видеоизображении компьютерной мыши (фон);

2) осуществление самостоятельных движений компьютерной мышью по кругу (Дв.2) (рис. 1В);

3) имитация детьми движений, выполняемых экспериментатором (имитация биологического движения, ИмБ) (рис. 1Г).

ЭЭГ анализировали в индивидуальном частотном диапазоне н-ритма, определяемого при совершении 
самостоятельных движений правой рукой в отведении С3. Полный частотный диапазон н-ритма (6-13 Гц) разбивали на отрезки по 1 Гц. В качестве индивидуального частотного диапазона принимали два прилегающих друг к другу отрезка с максимальной десинхронизацией относительно фона [17]. Амплитуду н-ритма в пределах индивидуального частотного диапазона рассчитывали для каждой экспериментальной ситуации. С целью нормализации распределения значения амплитуд логарифмировали.

Для сопоставления параметров н-ритма в ситуациях имитации биологического и небиологического движений использовали индексы реактивности. Указанные показатели рассчитывали по общепринятой схеме [18], в соответствии с фрормулой: $k=\ln (\mathrm{B} / \mathrm{A})$, где $k$ - индекс реактивности сенсомоторного ритма, В - амплитуда сенсомоторного ритма в основной ситуации, А 一 амплитуда сенсомоторного ритма в исходной ситуации сравнения (фон или самостоятельное движение). Положительные значения индекса реактивности соответствовали синхронизации сенсомоторного ритма, а отрицательные десинхронизации.

\section{Статистическая обработка данных}

Статистический анализ данных проводили с использованием пакета STATISTICA 12.0 (StatSoft Inc.; США). Для описания распределений, отличных от нормального, применяли медиану и интерквартильный размах, а различия между группами оценивали с помощью U-критерия МаннаУитни. В случаях нормального распределения данных использовали среднее и стандартную ошибку среднего. Различия в амплитуде и индексах реактивности н-ритма, зарегистрированного в разных экспериментальных ситуациях, оценивали с помощью дисперсионного анализа с повторными измерениями (repeated measures ANOVA). По схеме 4×2×9 оценивали влияние межсубъектного фактора - возрастной группы (ВОЗР), а также двух внутрисубъектных факторов - ситуации (СИТ) и локуса ЭЭГ (ЛОК). Для расчета статистической значимости различий в анализируемых параметрах сенсомоторного ритма в привязке к каждому из девяти отведений ЭЭГ в рамках каждой возрастной группы использовали метод априорных контрастов (оценку F-распределения).

\section{РЕЗУЛЬТАТЫ ИССЛЕДОВАНИЯ}

\section{Частотные показатели $\mu$-ритма}

Значения медианы нижней границы индивидуального диапазона н-ритма составили 9 Гц $(8,5 ; 10)$ при крайних значениях 6 и 11 Гц. Значения медианы верхней границы индивидуального диапазона н-ритма составили 11 Гц (10,5; 12) при крайних значениях 8 и 13 Гц. Различия по данному показателю между возрастными группами не достигали уровня статистической значимости.

\section{Амплитуда $\mu$-ритма ЭЭГ в состоянии двигательного покоя, а также при выполнении и имитации действий}

Дисперсионный анализ различий в амплитуде $\mu$-ритма в ситуации Дв.1, относительно фона, с учетом возрастной группы и локуса отведения ЭЭГ показал значимое влияние факторов СИТ $\left(\mathrm{F}_{1,132}=90,34 ; p<0,001\right)$, ВО3Р $\left(\mathrm{F}_{3,132}=\right.$ $10,18 ; p<0,001)$ и ЛОК $\left(F_{8,1056}=73,06 ; p<0,001\right)$, а также взаимодействия факторов СИТ×ЛОК $\left(\mathrm{F}_{8,1056}=41,28\right.$; p < 0,001). В ситуации ИмНБ, относительно Дв.1 на изменения амплитуды н-ритма значимо влияли факторы СИТ $\left(F_{1,132}=12,65 ; p<0,001\right)$, ВОЗР $\left(F_{3,132}=14,67\right.$; $p<0,001)$ и ЛОК $\left(F_{8,1056}=39,43 ; p<0,001\right)$.

Анализ различий в амплитуде $\mu$-ритма в ситуации Дв.2 относительно фона и с учетом возрастной группы и локуса отведения ЭЭГ показал значимое влияние факторов СИТ $\left(F_{1,132}=31,17 ; p<0,001\right)$, ВОЗР $\left(F_{3,132}=6,46 ; p<0,001\right)$ и ЛОК $\left(\mathrm{F}_{8,1056}=71,55 ; p<0,001\right)$, а также взаимодействия факторов СИТ $\times Л О К ~\left(F_{8,1056}=28,32 ; p<0,001\right)$ и СИТ×ВОЗР $\left(\mathrm{F}_{3,132}=6,35 ; p<0,001\right)$. При оценке изменения амплитуды н-ритма в ситуации ИмБ относительно Дв.2 было показано значимое влияние факторов СИТ $\left(F_{1,132}=9,58 ; p=0,002\right)$, BО3Р $\left(F_{3,132}=18,63 ; p<0,001\right)$ и ЛОК $\left(F_{8,1056}=54,08\right.$; $p<0,001)$, а также взаимодействия факторов СИТ×ЛОК $\left(\mathrm{F}_{8,1056}=3,28 ; p=0,001\right)$ и СИТ $\times$ ВОЗР $\left(\mathrm{F}_{3,132}=6,2 ; p=0,001\right)$.

У детей 4-6 лет при осуществлении концентрических перемещений цветного круга была выявлена значимая десинхронизация н-ритма ЭЭГ (Дв.1 относительно фона) в большинстве исследуемых областей. При имитации движений цветного круга изменения амплитуды н-ритма (ИмНБ относительно Дв.1) не достигали уровня статистической значимости (рис. 2А). При выполнении самостоятельных движений компьютерной мышью у детей данного возраста был зарегистрирован значимый рост амплитуды н-ритма ЭЭГ (Дв.2 относительно фона) в центральном локусе правого полушария (C4). При имитации движений экспериментатора была обнаружена десинхронизация сенсомоторного ритма (ИмБ относительно Дв. 2) в срединном теменном отведении (Pz) (рис. 2Б).

В группе детей 7-9 лет значимая депрессия $\mu$-ритма в ситуации Дв.1 была обнаружена в большинстве исследуемых областей. При ИмНБ относительно Дв.1
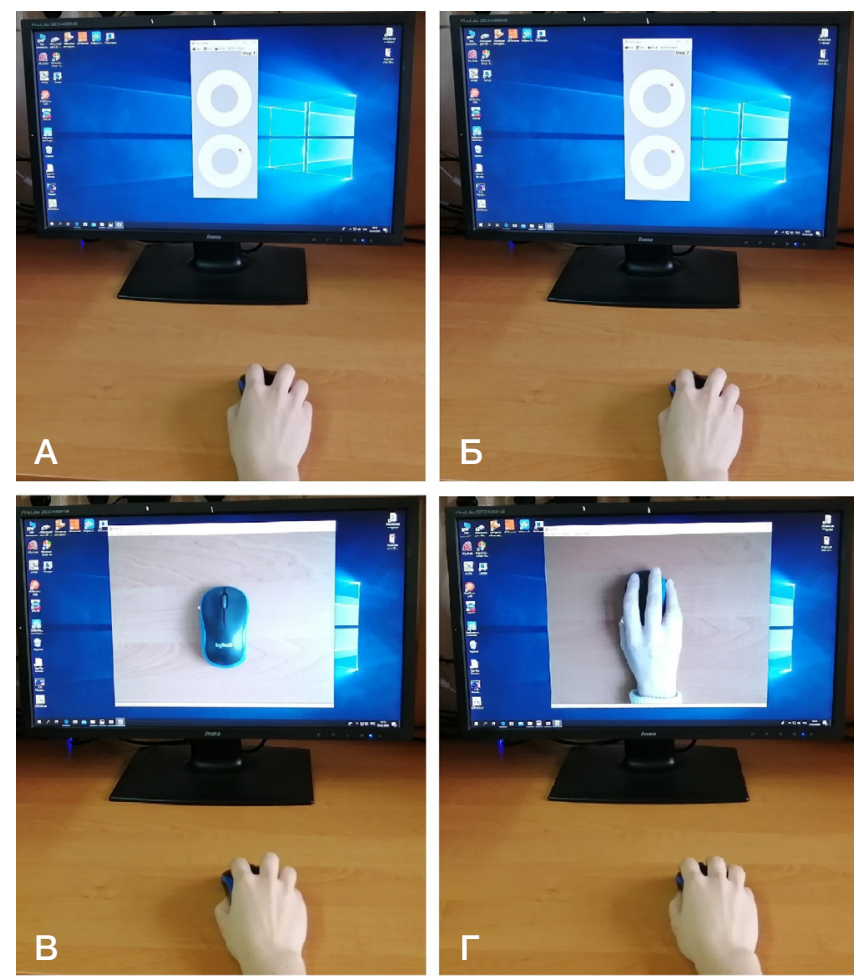

Рис. 1. Серия заданий. А. Осуществление концентрических перемещений цветного круга на экране монитора при помощи компьютерной мыши (Дв.1). Б. Имитация движений другого цветного круга (ИмНБ). В. Осуществление самостоятельных движений компьютерной мышью по кругу (Дв.2). Г. Имитация детьми движений, выполняемых экспериментатором (ИмБ) 
изменения амплитуды н-ритма не достигали уровня статистической значимости (рис. 3А). В ситуации Дв.2 значимая десинхронизация сенсомоторного ритма была обнаружена в центральных (C3 и Cz) и всех теменных локусах. При ИмБ относительно Дв.2 не выявлено значимых изменений амплитуды н-ритма (рис. ЗБ).

У детей 10-12 лет при Дв.1 значимое падение амплитуды $\mu$-ритма зарегистрировано в большинстве анализируемых локусов. При имитации движений цветного круга обнаружена значимая синхронизация сенсомоторного ритма (ИмНБ относительно Дв.1) в срединном фронтальном отведении (рис. 4А). В ситуации Дв.2 значимое падение амплитуды н-ритма наблюдали в центральных (C3 и Cz) и всех теменных локусах. При ИмБ относительно Дв.2 изменения амплитуды сенсомоторного ритма не достигали уровня статистической значимости (рис. 4Б).

В группе подростков 13-15 лет при Дв.1 снижение амплитуды $\mu$-ритма достигало уровня статистической значимости во всех анализируемых локусах. При имитации небиологического движения в большинстве исследуемых областей была зарегистрирована значимая синхронизация (ИмНБ, относительно Дв.1) сенсомоторного ритма (рис. 5A). При выполнении самостоятельных движений компьютерной мышью значимая десинхронизация сенсомоторного ритма (Дв.2 относительно фона) была обнаружена во всехисследуемых областях. В ситуации ИмБ выявлена дополнительная, по сравнению с предыдущим заданием, десинхронизация н-ритма, значимая во всех отведениях (рис. 5Б).

\section{Сравнение реактивности $\mu$-ритма ЭЭГ при имитации движений визуальных образов биологического и небиологического происхождения}

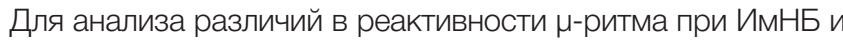
ИмБ, относительно условий выполнения самостоятельных движений с произвольной скоростью, был проведен дисперсионный анализ индексов реактивности с учетом

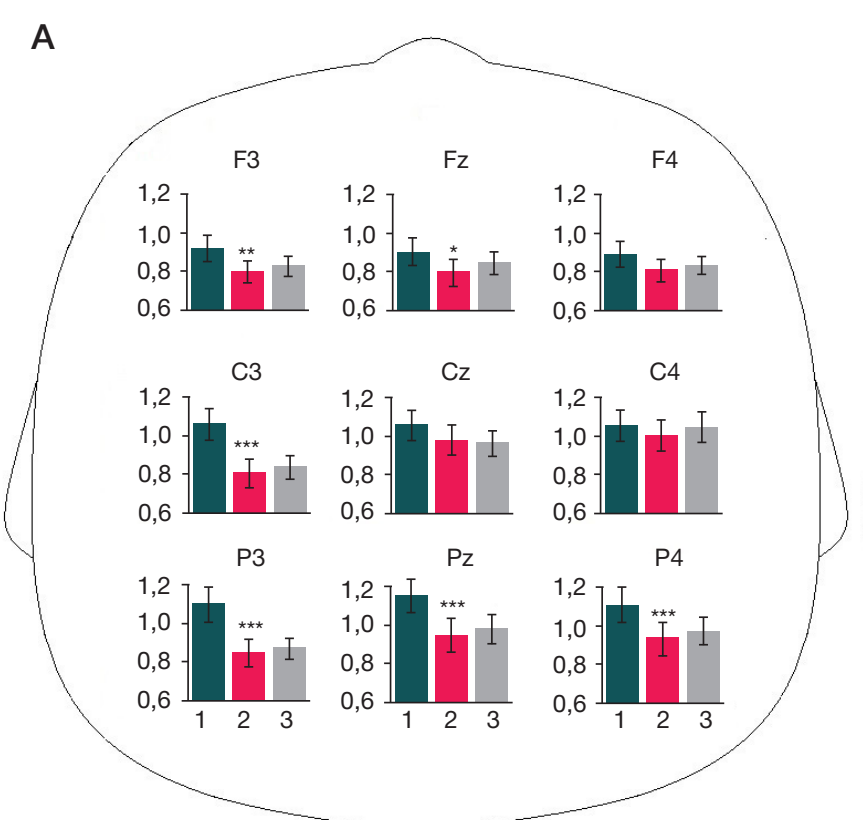

возрастной группы и локуса отведения ЭЭГ. Средние значения индексов реактивности н-ритма у детей в четырех возрастных группах представлены в табл. 1 и 2. Выявлено значимое влияние факторов СИТ ( $\left.F_{1,132}=21,85 ; p<0,001\right)$ и ЛОК $\left(F_{8,1056}=3,95 ; p<0,001\right)$, а также взаимодействия факторов СИТ×ВОЗР $\left(\mathrm{F}_{3,132}=5,52 ; p=0,001\right)$. В группе детей дошкольного возраста различия в индексах реактивности $\mu$-ритма ЭЭГ при ИмНБ и ИмБ достигали уровня статистической значимости в теменных отведениях Pz и P4 ( $p=0,03)$. У детей 7-9 лет анализируемые различия были статистически значимыми для локуса Fz ( $p=0,04)$. У детей 10-12 лет различия в индексах реактивности н-ритма не достигали уровня статистической значимости. В группе подростков 13-15 лет анализируемые различия были статистически значимыми во всех исследуемых областях $(p \leq 0,001)$.

\section{ОБСУЖДЕНИЕ РЕЗУЛЬТАТОВ}

Результаты исследования показали, что индивидуальные границы частотного диапазона сенсомоторного ритма у детей 4-15 лет широко варьируют, а их средние величины значимо не различаются у разных возрастных групп. В работе других авторов [19], посвященной анализу реактивности н-ритма ЭЭГ в индивидуально определенном частотном диапазоне у детей 4-11 лет, полоса сенсомоторного ритма также составляла в среднем 9-11 Гц, были отмечены высокая межиндивидуальная вариативность его параметров и отсутствие связи с возрастом детей. Все это указывает на необходимость определения индивидуального частотного диапазона у детей при проведении исследований реактивности сенсомоторного ритма, а также перед проведением коррекционных мероприятий с использованием параметров н-ритма (тренингов на основе биологической обратной связи по ЭЭГ, применения интерфейсов мозгкомпьютер).

Анализ изменений амплитуды н-ритма показал, что у детей 4-6 лет при выполнении произвольных

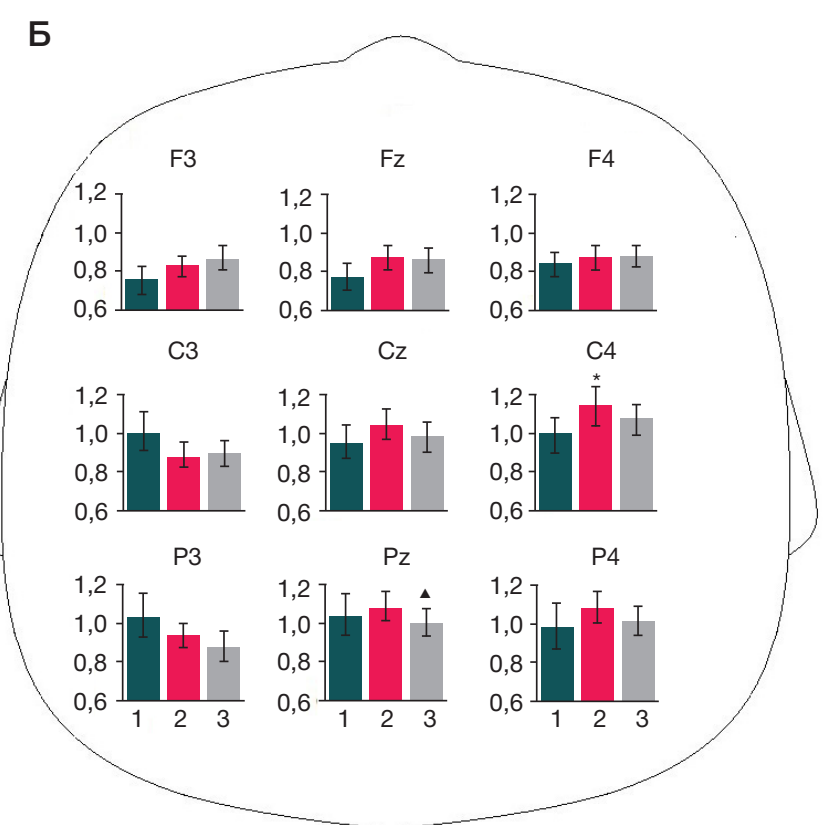

Рис. 2. Амплитуда (A, Ln мkB) н-ритма ЭЭг у детей 4-6 лет при имитации движений визуальных образов небиологического (А) и биологического (Б) происхождения. 1 - фон, 2 - выполнение самостоятельных движений с произвольной скоростью, 3 - имитация движений. Различия амплитуд в фоне и при выполнении самостоятельных движений: * $-p<0,05 ;{ }^{* *}-p \leq 0,01 ;{ }^{* \star \star}-p \leq 0,001 ;$ при выполнении самостоятельных движений и имитации: $\Delta-p<0,05$ 
перемещений цветного круга (Дв.1) можно наблюдать значимую десинхронизацию $\mu$-ритма ЭЭГ во фронтальном и центральном отведениях левого полушария, медианном фронтальном отведении и всех теменных областях (F3, Fz, C3, P3, Pz, P4). Полученные в настоящем исследовании результаты согласуются с уже имеющимися в литературе данными о десинхронизации сенсомоторного ритма при осуществлении произвольных движений [20]. При выполнении самостоятельных движений компьютерной мышью (Дв.2) значимого падения амплитуды н-ритма не наблюдалось. Возможно, что для детей данного возраста задание на выполнение относительно простых круговых движений компьютерной мышью было проще задания на захват и перемещение цветного круга при помощи левой кнопки компьютерной мыши и не требовало особого двигательного контроля. При выполнении этого задания был также выявлен значимый рост амплитуды н-ритма

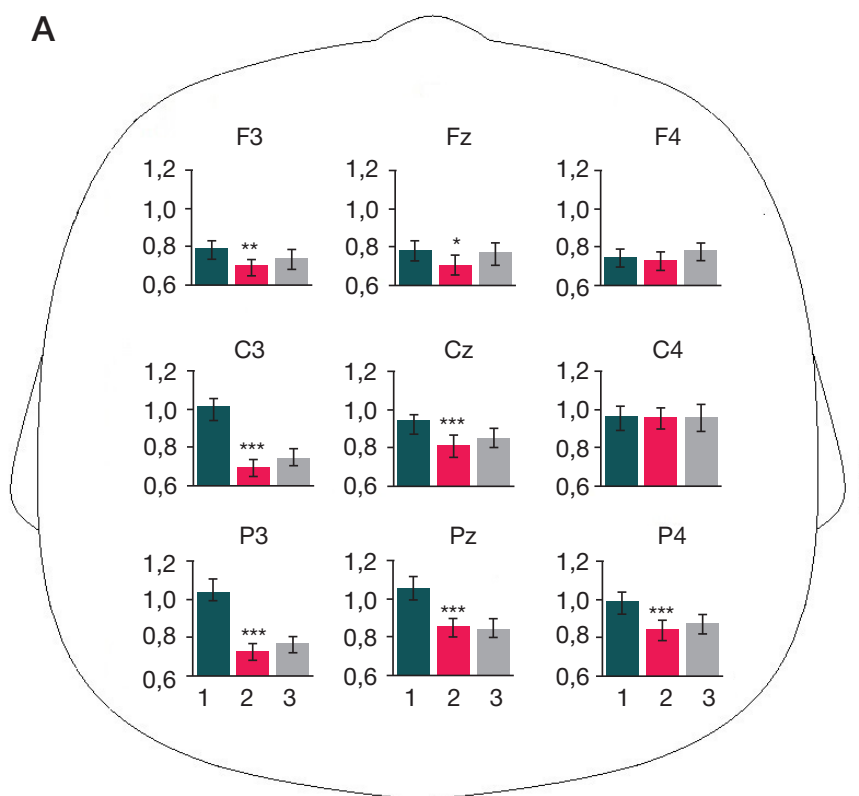

ЭЭГ в локусе C4, что может быть связано с торможением в ипсилатеральном, относительно задействованной руки, полушарии [21].

В группах детей 7-9 и 10-12 лет десинхронизация н-ритма при выполнении самостоятельных движений была обнаружена в большинстве исследуемых областей. При этом условие осуществления концентрических перемещений цветного круга (Дв.1), в отличие от условия выполнения движений мышью по кругу (Дв.2), также сопровождалось десинхронизацией сенсомоторного ритма во фрронтальных локусах (F3, Fz). Известно, что лобные области коры головного мозга осуществляют планирование и подготовку сложных движений [22]. Предполагается также, что более сложные двигательные акты сопровождаются более широко распространенной десинхронизацией предположить, что выполнение перемещений цветного

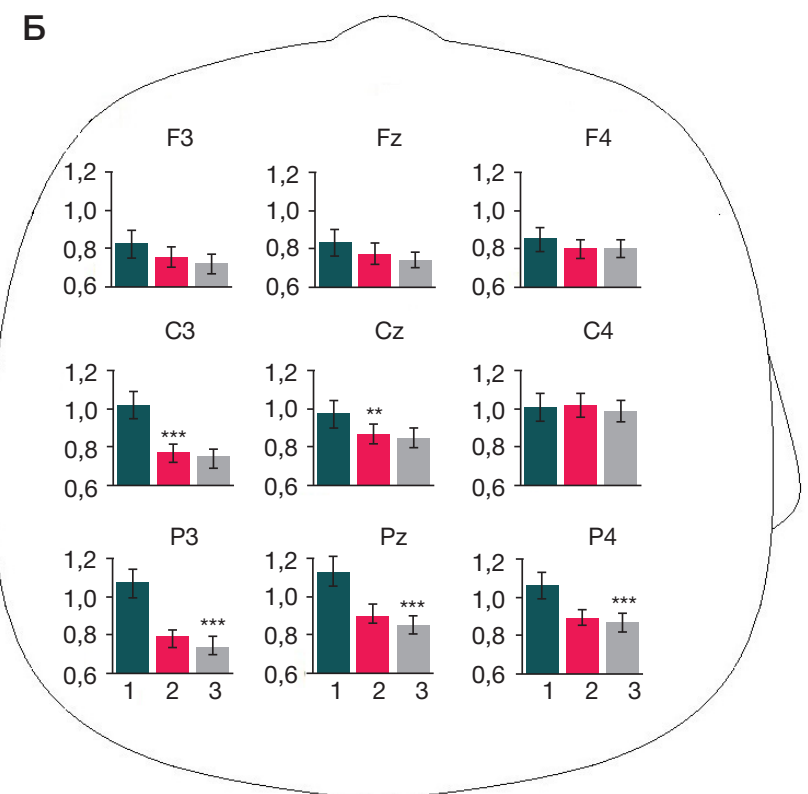

Рис. 3. Амплитуда (A, Ln MKB) н-ритма ЭЭГ в группе детей 7-9 лет при имитации движений визуальных образов небиологического (А) и биологического (Б) происхождения. Остальные обозначения те же, что на рис. 2

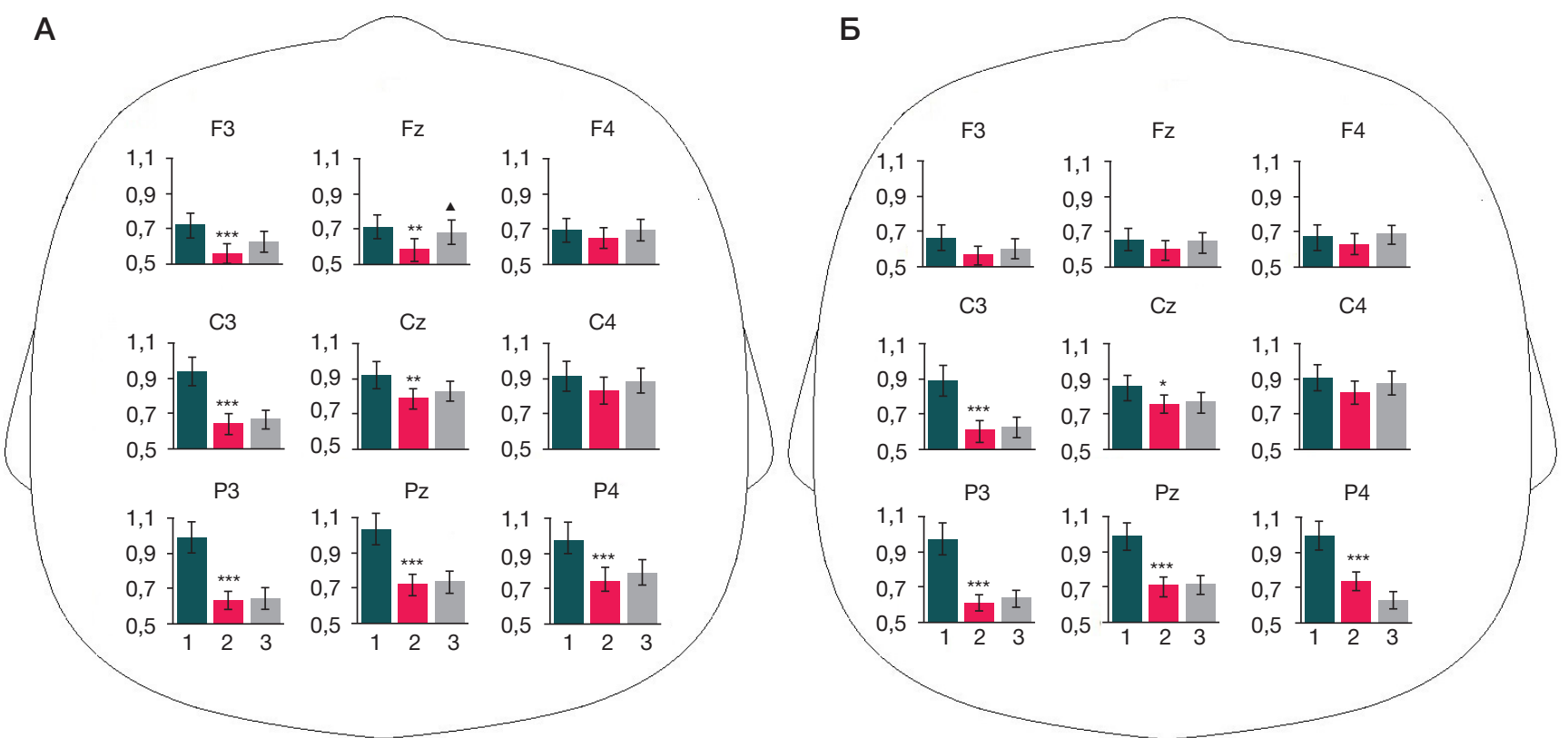

Рис. 4. Амплитуда (A, Ln мкB) н-ритма ЭЭГ в группе детей 10-12 лет при имитации движений визуальных образов небиологического (А) и биологического (Б) происхождения. Остальные обозначения те же, что на рис. 2 
круга у детей 7-12 лет, как и у младшей группы, требовало значительных усилий, что и приводило к вовлечению фронтальных областей коры.

Ситуации имитации движений визуальных образов биологического и небиологического происхождения у детей 4-6, 7-9 и 10-12 лет почти не сопровождались дополнительной модуляцией $\mu$-ритма относительно выполнения произвольных движений. Выявленный факт может свидетельствовать о том, что у детей данного возраста в условиях подражания дополнительные ресурсы неокортекса, требуемые для переработки разномодальной информации, вовлекаются недостаточно.

В группе подростков 13-15 лет значимая десинхронизация сенсомоторного ритма при выполнении самостоятельных движений была обнаружена во всех исследуемых областях. Снижение амплитуды н-ритма над фронтальными, центральными и париетальными локусами у детей старшего возраста может быть обусловлено развитием связей между задействованными областями неокортекса. При имитации движений визуальных образов небиологического происхождения наблюдалось меньшее падение амплитуды сенсомоторного ритма, чем при выполнении произвольных движений и подражании движениям визуальных образов биологического происхождения (что особенно заметно во фронтальных и центральных локусах). Можно предположить, что необходимость подражать движениям другого объекта (цветного круга) привела к переключению внимания на его восприятие и, как следствие, к ослаблению моторного контроля собственных движений. У детей данного возраста в ситуации ИмБ выявлена дополнительная, по сравнению с наблюдаемой при выполнении задания Дв.2, значимая десинхронизация н-ритма во всех отведениях. Обращает на себя внимание наибольшая выраженность такой реакции в париетальных локусах. Известно, что париетальные области коры больше вовлечены в переработку информации при наблюдении движений, выполняемых человеком, в сравнении с наблюдением движений небиологических объектов [24]. Выявленная в настоящем исследовании модуляция сенсомоторного ритма в данных областях при подражании движениям визуальных образов биологического происхождения может указывать на участие находящихся в теменной коре компонентов СЗН, связанных с кодированием целей, лежащих в основе наблюдаемых действий [25]. Зеркальные нейроны связывают с когнитивной интеграцией визуальных, слуховых и моторных стимулов для обеспечения социального взаимодействия у детей [26] и взрослых [27]. В связи с этим, можно предположить, что дополнительная десинхронизация н-ритма при имитации движений другого человека вызвана именно наличием социального контекста, к которому чувствительна СЗН.

При сравнении индексов реактивности н-ритма в заданиях имитации было выявлено, что подражание движениям визуальных образов биологического происхождения у детей старшей группы сопровождается большей десинхронизацией, в сравнении с ситуацией подражания движениям цветного круга. Как уже отмечалось, подобные особенности реактивности сенсомоторного ритма во время наблюдения за движениями биологических и небиологических объектов выявлены у взрослых волонтеров [8].

Сравнительный анализ паттернов реактивности сенсомоторного ритма у детей разного возраста позволяет прийти к следующим заключениям. В группе младших детей (4-6 лет) наибольшая активация фронтальных, центральных и париетальных областей коры мозга, проявляющаяся в снижении амплитуды н-ритма (более значимого в левом полушарии), наблюдается при движениях компьютерной мышью, ассоциированных с небиологическим объектом (цветной круг) (Дв.1). Выполнение движений компьютерной мышью в произвольном ритме (Дв.2) не приводит к значимому снижению амплитуды н-ритма, а имитация биологического движения (движения руки другого человека) в большинстве отведений не сопровождается дополнительной активацией. Таким образом, в нашей экспериментальной ситуации у детей дошкольного возраста корковый отдел двигательного анализатора наиболее чувствителен к манипуляциям с небиологическими объектами. У детей более старших возрастных групп (7-9 и 10-12 лет) выявлен
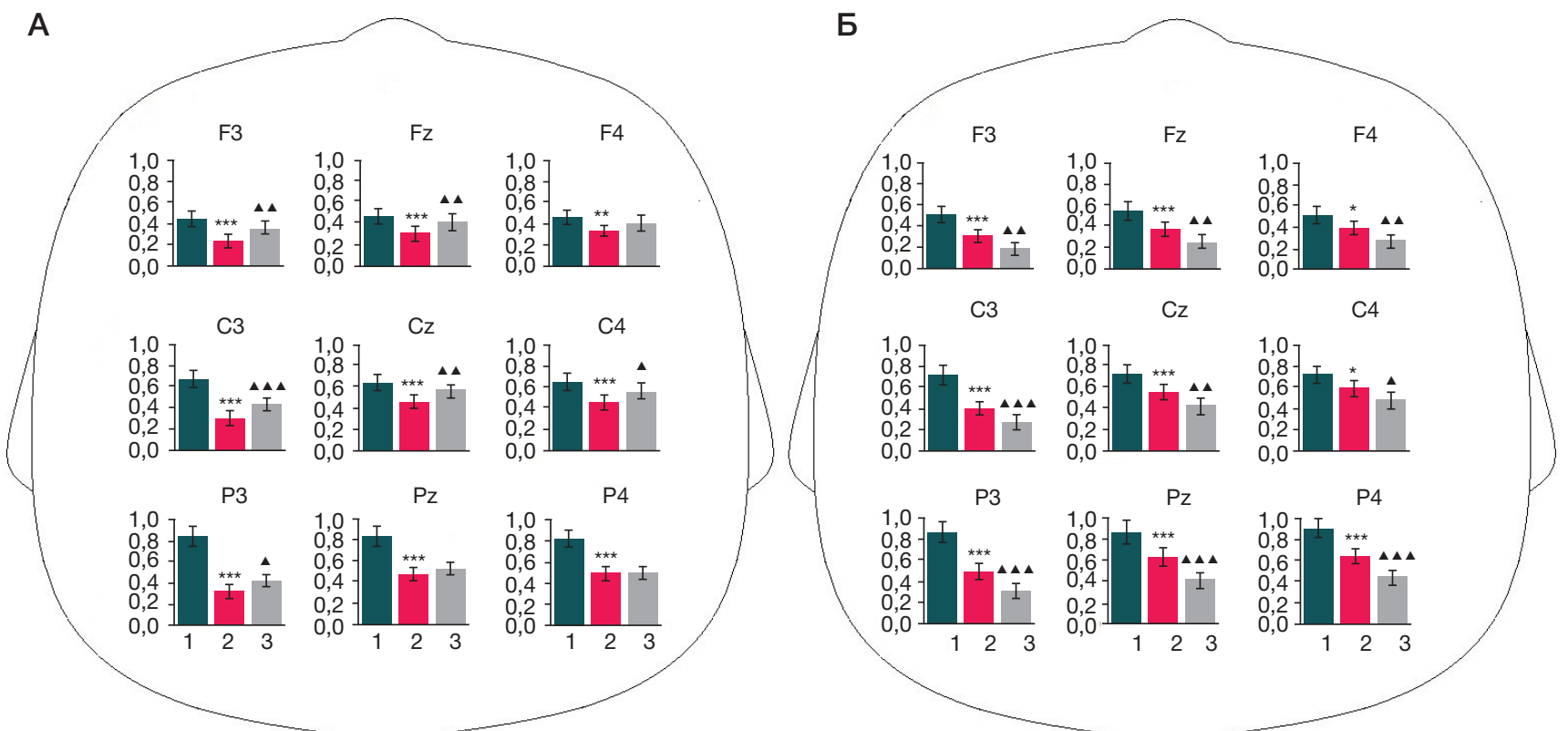

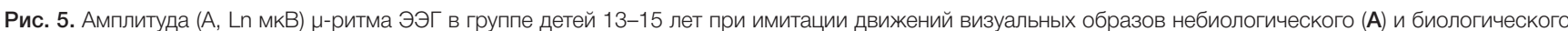
(Б) происхождения. Различия амплитуд при выполнении самостоятельных движений и имитации: ${ }^{\mathbf{\Delta}}-p \leq 0,01 ; \mathbf{\Delta \wedge}-p \leq 0,001$. Остальные обозначения те же, что на рис. 2 
Таблица 1. Средние значения индексов реактивности (с обозначением ошибки среднего) при имитации движений визуальных образов небиологического происхождения

\begin{tabular}{|c|c|c|c|c|c|c|c|c|c|}
\hline \multirow{2}{*}{ Группа } & \multicolumn{9}{|c|}{ Отведения ЭЭГ } \\
\cline { 2 - 10 } & $\mathrm{F} 3$ & $\mathrm{Fz}$ & $\mathrm{F} 4$ & $\mathrm{C} 3$ & $\mathrm{Cz}$ & $\mathrm{C} 4$ & $\mathrm{P} 3$ & $\mathrm{Pz}$ & $\mathrm{P} 4$ \\
\hline 4-6 лет & $0,03 \pm 0,04$ & $0,04 \pm 0,04$ & $0,03 \pm 0,03$ & $0,03 \pm 0,04$ & $-0,02 \pm 0,04$ & $0,04 \pm 0,03$ & $0,02 \pm 0,04$ & $0,03 \pm 0,04$ & $0,04 \pm 0,04$ \\
\hline $7-9$ лет & $0,05 \pm 0,03$ & $0,06 \pm 0,03$ & $0,05 \pm 0,03$ & $0,05 \pm 0,03$ & $0,04 \pm 0,03$ & $0,00 \pm 0,05$ & $0,04 \pm 0,03$ & $-0,01 \pm 0,03$ & $0,03 \pm 0,03$ \\
\hline $10-12$ лет & $0,06 \pm 0,04$ & $0,09 \pm 0,04$ & $0,05 \pm 0,04$ & $0,03 \pm 0,04$ & $0,04 \pm 0,03$ & $0,06 \pm 0,03$ & $0,01 \pm 0,04$ & $0,01 \pm 0,04$ & $0,05 \pm 0,05$ \\
\hline $13-15$ лет & $0,11 \pm 0,04$ & $0,11 \pm 0,05$ & $0,07 \pm 0,04$ & $0,12 \pm 0,03$ & $0,10 \pm 0,03$ & $0,11 \pm 0,04$ & $0,09 \pm 0,03$ & $0,05 \pm 0,03$ & $0,01 \pm 0,03$ \\
\hline
\end{tabular}

Примечание: положительные значения индекса реактивности соответствуют синхронизации сенсомоторного ритма, отрицательные значения — десинхронизации.

Таблица 2. Средние значения индексов реактивности (с обозначением ошибки среднего) при имитации движений визуальных образов биологического происхождения

\begin{tabular}{|c|c|c|c|c|c|c|c|c|c|}
\hline \multirow{2}{*}{ Группа } & \multicolumn{7}{|c|}{ Отведения ЭЭГ } \\
\cline { 2 - 10 } & $\mathrm{F} 3$ & $\mathrm{Fz}$ & $\mathrm{F} 4$ & $\mathrm{C} 3$ & $\mathrm{Cz}$ & $\mathrm{C} 4$ & $\mathrm{P} 3$ & $\mathrm{Pz}$ & $\mathrm{P} 4$ \\
\hline $4-6$ лет & $0,04 \pm 0,04$ & $-0,01 \pm 0,05$ & $0,01 \pm 0,05$ & $0,01 \pm 0,04$ & $-0,07 \pm 0,05$ & $-0,07 \pm 0,06$ & $-0,06 \pm 0,05$ & $-0,09 \pm 0,05$ & $-0,06 \pm 0,06$ \\
\hline $7-9$ лет & $-0,03 \pm 0,03$ & $-0,04 \pm 0,03$ & $0,00 \pm 0,03$ & $-0,03 \pm 0,02$ & $-0,03 \pm 0,03$ & $-0,03 \pm 0,04$ & $-0,04 \pm 0,04$ & $-0,06 \pm 0,03$ & $-0,03 \pm 0,03$ \\
\hline $10-12$ лет & $0,04 \pm 0,05$ & $0,04 \pm 0,04$ & $0,06 \pm 0,04$ & $0,03 \pm 0,03$ & $0,01 \pm 0,03$ & $0,06 \pm 0,04$ & $0,03 \pm 0,03$ & $0,01 \pm 0,04$ & $0,05 \pm 0,03$ \\
\hline $13-15$ лет & $-0,11 \pm 0,03$ & $-0,12 \pm 0,03$ & $-0,11 \pm 0,03$ & $-0,14 \pm 0,03$ & $-0,12 \pm 0,04$ & $-0,11 \pm 0,04$ & $-0,18 \pm 0,03$ & $-0,21 \pm 0,04$ & $-0,20 \pm 0,03$ \\
\hline
\end{tabular}

схожий паттерн активации неокортекса при движениях, ассоциированных с небиологическим объектом, при собственных движениях компьютерной мышью, а также при имитации движений биологического объекта (руки экспериментатора). В отличие от уже рассмотренных групп, испытуемые 13-15 лет демонстрируют статистически значимую десинхронизацию н-ритма во фронтальных, центральных и теменных областях коры обоих полушарий при имитации движений другого человека.

Логично предположить, что процессы восприятия и имитации движений других людей у детей младших возрастных групп находятся на стадии становления, в то время, как у подростков они достаточно развиты и схожи с таковыми у взрослых. Выраженная десинхронизация $\mu$-ритма у подростков при совершении самостоятельных движений и имитации движений визуальных образов биологического происхождения во всех исследуемых областях может быть обусловлена созреванием моторных, сенсомоторных и ассоциативных областей коры, вовлеченных в выполнение и имитацию действий [28]. Выявленные в настоящем исследовании возрастные особенности реактивности сенсомоторного ритма могут быть использованы при совершенствовании уже имеющихся коррекционных методик реабилитации детей с
ДЦП на основе роботизированных устройств, управляемых по характеристикам ЭЭГ [15].

\section{ВЫВОДЫ}

При выполнении детьми 4-15 лет самостоятельных движений компьютерной мышью развивается десинхронизация н-ритма во фронтальных, центральных и париетальных областях неокортекса, более выраженная в левом полушарии. При имитации движений визуальных образов биологического и небиологического происхождения у детей 4-6, 7-9 и 10-12 лет выявлена лишь незначительная, по сравнению с заданием на выполнение самостоятельных движений, дополнительная модуляция н-ритма. У детей 13-15 лет наибольшая десинхронизация сенсомоторного ритма отмечена в ситуации подражания движениям руки экспериментатора. При разработке программного обеспечения интерфейсов мозг-компьютер, предназначенных для коррекции двигательных расстройств, в качестве зрительных стимулов могут быть использованы элементы движений небиологических объектов, но чем старше дети, тем более эффективным для активации неокортекса может быть предъявление движущихся биологических объектов.

\section{Литература}

1. Rizzolatti G, Fogassi L. The mirror mechanism: recent findings and perspectives. Philos Trans R Soc Lond B Biol Sci. 2014; 369 (1644): 20130420

2. Hardwick RM, Caspers S, Eickhoff SB, Swinnen SP. Neural correlates of action: Comparing meta-analyses of imagery, observation, and execution. Neurosci Biobehav Rev. 2018; (94): $31-44$.

3. Лебедева Н. Н., Зусмман А. И., Мальцев В. Ю. Система зеркальных нейронов мозга: ключ К обучению, формированию личности и пониманию чужого сознания. Успехи физиологических наук. 2017; 48 (4): 16-28.

4. Fox NA, Bakermans-Kranenburg MJ, Yoo KH, Bowman LC Cannon EN, Vanderwert RE, et al. Assessing human mirror activity with EEG mu rhythm: A meta-analysis. Psychol Bull. 2016; 142 (3): 291-313.

5. Hobson HM, Bishop DVM. Mu suppression - a good measure of the human mirror neuron system? Cortex. 2016; (82): 290-310.

6. Gundlach C, Muller MM, Nierhaus T, Villringer A, Sehm B. Modulation of Somatosensory Alpha Rhythm by Transcranial Alternating Current Stimulation at Mu-Frequency. Front Hum Neurosci. 2017; (11): 432.

7. Bimbi M, Festante F, Coude G, Vanderwert RE, Fox NA, Ferrari PF. Simultaneous scalp recorded EEG and local field potentials from monkey ventral premotor cortex during action observation and execution reveals the contribution of mirror and motor neurons to the mu-rhythm. Neuroimage. 2018; (175): 22-31.

8. Ulloa ER, Pineda JA. Recognition of point-light biological motion: mu rhythms and mirror neuron activity. Behav Brain Res. 2007; 183 (2): 188-94.

9. Oberman LM, Pineda JA, Ramachandran VS. The human mirror 
neuron system: a link between action observation and social skills. Soc Cogn Affect Neurosci. 2007; 2 (1): 62-66.

10. Wohlschlager $A$, Gattis $M$, Bekkering $H$. Action generation and action perception in imitation: an instance of the ideomotor principle. Philos. Trans. R Soc. Lond., B Biol Sci. 2003; (358): 501-15.

11. Фролов А. А., Бобров П. Д. Интерфейс мозг-компьютер: нейрофизиологические предпосылки и клиническое применение. Журнал высшей нервной деятельности. 2017; 67 (4): 365-76.

12. Левицкая О. С., Лебедев М. А. Интерфейс мозгкомпьютер: будущее в настоящем. Вестник Российского Государственного Медицинского Университета. 2016; (2): 4-16.

13. Lopez-Larraz E, Escolano C, Montesano L, Minguez J. Reactivating the Dormant Motor Cortex After Spinal Cord Injury With EEG Neurofeedback: A Case Study With a Chronic, Complete C4 Patient. Clin EEG Neurosci. 2019; 50 (2): 100-10.

14. Либуркина С. П., Васильев А. Н., Каплан А. Я., Иванова Г. Е., Чуканова А. С. Пилотное исследование идеомоторного тренинга в контуре интерфейса мозг-компьютер у пациентов с двигательными нарушениями. Журнал неврологии и психиатрии. 2018; 9 (2): 63-68.

15. Ларина Н. В., Корсунская Л. Л., Власенко С. В. Комплекс «Экзокисть-2» в реабилитации верхней конечности при детском церебральном параличе с использованием неинвазивного интерфейса «мозг-компьютер». Нервномышечные болезни. 2019; 9 (4): 44-50.

16. Cannon EN, Yoo KH, Vanderwert RE, Ferrari PF, Woodward AL, Fox NA. Action experience, more than observation, influences mu rhythm desynchronization. PLoS One. 2014; 9 (3): e92002.

17. Махин С. А., Кайда А. И., Эйсмонт Е. В., Михайлова А. А., Павленко В. Б.; ФГАОУ ВО «Крымский федеральный университет имени В. И. Вернадского», патентообладатель. Способ определения индивидуального частотного диапазона н-ритма ЭЭГ. Патент РФ № 2702728. 09.10.2019.

\section{References}

1. Rizzolatti G, Fogassi $L$. The mirror mechanism: recent findings and perspectives. Philos Trans R Soc Lond B Biol Sci. 2014; 369 (1644): 20130420.

2. Hardwick RM, Caspers S, Eickhoff SB, Swinnen SP. Neural correlates of action: Comparing meta-analyses of imagery, observation, and execution. Neurosci Biobehav Rev. 2018; (94): $31-44$.

3. Lebedeva NN, Zufman Al, Mal'cev VJu. Sistema zerkal'nyh nejronov mozga: kljuch $\mathrm{k}$ obucheniju, formirovaniju lichnosti ponimaniju chuzhogo soznanija. Uspehi fiziologicheskih nauk. 2017; 48(4): 16-28.

4. Fox NA, Bakermans-Kranenburg MJ, Yoo KH, Bowman LC, Cannon EN, Vanderwert RE, et al. Assessing human mirror activity with EEG mu rhythm: A meta-analysis. Psychol Bull. 2016; 142 (3): 291-313.

5. Hobson HM, Bishop DVM. Mu suppression - a good measure of the human mirror neuron system? Cortex. 2016; (82): 290-310.

6. Gundlach C, Muller MM, Nierhaus T, Villringer A, Sehm B. Modulation of Somatosensory Alpha Rhythm by Transcranial Alternating Current Stimulation at Mu-Frequency. Front Hum Neurosci. 2017; (11): 432.

7. Bimbi M, Festante F, Coude G, Vanderwert RE, Fox NA, Ferrari PF. Simultaneous scalp recorded EEG and local field potentials from monkey ventral premotor cortex during action observation and execution reveals the contribution of mirror and motor neurons to the mu-rhythm. Neuroimage. 2018; (175): 22-31.

8. Ulloa ER, Pineda JA. Recognition of point-light biological motion: mu rhythms and mirror neuron activity. Behav Brain Res. 2007; 183 (2): 188-94.

9. Oberman LM, Pineda JA, Ramachandran VS. The human mirror neuron system: a link between action observation and social skills. Soc Cogn Affect Neurosci. 2007; 2 (1): 62-66.
18. Raymaekers R, Wiersema JR, Roeyers H. EEG study of the mirror neuron system in children with high functioning autism. Brain research. 2009; (1304): 113-21.

19. Lepage JF, Théoret H. EEG evidence for the presence of an action observation-execution matching system in children. Eur $\mathrm{J}$ Neurosci. 2006; 23 (9): 2505-10.

20. Лебедева Н. Н., Каримова Е. Д., Карпычев В. В., Мальцев В. Ю. Зеркальная система мозга при наблюдении, выполнении и представлении моторных задач - нейросизиологическое отражение восприятия чужого сознания. Журнал высшей нервной деятельности. 2018; 68 (2): 204-15.

21. Hummel $F$, Andres F, Altenmüller E, Dichgans J, Gerloff $C$. Inhibitory control of acquired motor programmes in the human brain. Brain. 2002; 125 (2): 404-20.

22. Brown MN, Staines WR. Differential effects of continuous theta burst stimulation over left premotor cortex and right prefrontal cortex on modulating upper limb somatosensory input. Neuroimage. 2016; (127): 97-109.

23. Thorpe SG, Cannon EN, Fox NA. Spectral and source structural development of $\mathrm{mu}$ and alpha rhythms from infancy through adulthood. Clin Neurophysiol. 2016; 127 (1): 254-69.

24. Saygin AP, Stadler W. The role of appearance and motion in action prediction. Psychol Res. 2012; (76): 388-94.

25. Bonini L, Rozzi S, Serventi FU, Simone L, Ferrari PF, Fogassi L. Ventral premotor and inferior parietal cortices make distinct contribution to action organization and intention understanding. Cereb Cortex. 2010; (20): 1372-85.

26. Filippi CA, Cannon EN, Fox NA, Thonpe SG, Ferrari PF, Woodward AL. Motor system activation predicts goal imitation in 7-month-old infants. Psychol Sci. 2016; (27): 675-84.

27. Yin J, Ding $X, X u H$, Zhang F, Shen M. Social Coordination Information in Dynamic Chase Modulates EEG Mu Rhythm. Sci Rep. 2017; 7 (1): 4782.

28. Segalowitz SJ, Santesso DL, Jetha MK. Electrophysiological changes during adolescence: a review. Brain Cogn. 2010; 72 (1): 86-100.

10. Wohlschlager A, Gattis $\mathrm{M}$, Bekkering $\mathrm{H}$. Action generation and action perception in imitation: an instance of the ideomotor principle. Philos. Trans. R Soc. Lond., B Biol Sci. 2003; (358): $501-15$.

11. Frolov AA, Bobrov PD. Interfejs mozg-komp'juter: nejrofiziologicheskie predposylki i klinicheskoe primenenie. Zhurnal vysshej nervnoj dejatel'nosti. 2017; 67(4): 365-376.

12. Levickaja OS, Lebedev MA. Interfejs mozg-komp'juter: budushhee $\checkmark$ nastojashhem. Vestnik Rossijskogo Gosudarstvennogo Medicinskogo Universiteta. 2016; (2): 4-16.

13. Lopez-Larraz E, Escolano C, Montesano L, Minguez J. Reactivating the Dormant Motor Cortex After Spinal Cord Injury With EEG Neurofeedback: A Case Study With a Chronic, Complete C4 Patient. Clin EEG Neurosci. 2019; 50 (2): 100-10.

14. Liburkina SP, Vasilev AN, Kaplan AJa, Ivanova GE, Chukanova AS. Pilotnoe issledovanie ideomotornogo treninga $v$ konture interfejsa mozg-komp'juter u pacientov $s$ dvigatel'nymi narushenijami. Zhurnal nevrologii i psihiatrii. 2018; 9 (2): 63-68.

15. Larina NV, Korsunskaja LL, Vlasenko SV. Kompleks «Jekzokist'-2» v reabilitacii verhnej konechnosti pri detskom cerebral'nom paraliche $s$ ispol'zovaniem neinvazivnogo interfejsa «mozg-komp'juter». Nervno-myshechnye bolezni. 2019; 9 (4): 44-50.

16. Cannon EN, Yoo KH, Vanderwert RE, Ferrari PF, Woodward AL, Fox NA. Action experience, more than observation, influences mu rhythm desynchronization. PLoS One. 2014; 9 (3): e92002.

17. Mahin SA, Kaida Al, Eismont EV, Mihailova AA, Pavlenko VB; FGAOU VO «Krymskij federal'nyj universitet imeni V.I. Vernadskogo», patentoobladatel'. Sposob opredelenija individual'nogo chastotnogo diapazona mju-ritma JeJeG. Patent RF № 2702728. 09.10.2019.

18. Raymaekers R, Wiersema JR, Roeyers H. EEG study of the mirror neuron system in children with high functioning autism. Brain 
research. 2009; (1304): 113-21.

19. Lepage JF, Théoret H. EEG evidence for the presence of an action observation-execution matching system in children. Eur $J$ Neurosci. 2006; 23 (9): 2505-10.

20. Lebedeva NN, Karimova ED, Karpychev VV, Malcev VJu Zerkal'naja sistema mozga pri nabljudenii, vypolnenii i predstavlenil motornyh zadach - nejrofiziologicheskoe otrazhenie vosprijatija chuzhogo soznanija. Zhurnal vysshej nervnoj dejatel'nosti. 2018; 68 (2): 204-15.

21. Hummel F, Andres F, Altenmüller E, Dichgans J, Gerloff C. Inhibitory control of acquired motor programmes in the human brain. Brain. 2002; 125 (2): 404-20.

22. Brown MN, Staines WR. Differential effects of continuous theta burst stimulation over left premotor cortex and right prefrontal cortex on modulating upper limb somatosensory input. Neuroimage. 2016; (127): 97-109.

23. Thorpe SG, Cannon EN, Fox NA. Spectral and source structural development of mu and alpha rhythms from infancy through adulthood. Clin Neurophysiol. 2016; 127 (1): 254-69.

24. Saygin AP, Stadler W. The role of appearance and motion in action prediction. Psychol Res. 2012; (76): 388-94.

25. Bonini L, Rozzi S, Serventi FU, Simone L, Ferrari PF, Fogassi L. Ventral premotor and inferior parietal cortices make distinct contribution to action organization and intention understanding. Cereb Cortex. 2010; (20): 1372-85.

26. Filippi CA, Cannon EN, Fox NA, Thorpe SG, Ferrari PF, Woodward AL. Motor system activation predicts goal imitation in 7-month-old infants. Psychol Sci. 2016; (27): 675-84.

27. Yin J, Ding $X, X u H$, Zhang $F$, Shen $M$. Social Coordination Information in Dynamic Chase Modulates EEG Mu Rhythm. Sci Rep. 2017; 7 (1): 4782.

28. Segalowitz SJ, Santesso DL, Jetha MK. Electrophysiological changes during adolescence: a review. Brain Cogn. 2010; 72 (1): 86-100. 